\title{
Integrating Diabetes Prevention Education Among Teenagers Involved in Summer Employment: Encouraging Environments for Health in Adolescence (ENHANCE)
}

\author{
Lisa Yazel-Smith, MS, MCHES (corresponding author) \\ Research Coordinator \\ Pediatric and Adolescent Comparative Effectiveness Research \\ Indiana University School of Medicine, Department of Pediatrics \\ $410 \mathrm{~W} .10^{\text {th }}$ Street, Suite 2000A, Indianapolis, IN 46202 \\ $\mathrm{Ph}$ (317) 278-9615, F (317) 321-0128 \\ smithlg@iupui.edu
}

Author contribution: first draft of manuscript; study coordination

Hala K. El-Mikati, M.D.

Research Associate

Pediatric and Adolescent Comparative Effectiveness Research

Indiana University School of Medicine, Department of Pediatrics

$410 \mathrm{~W} .10^{\text {th }}$ Street, Suite 2000A, Indianapolis, IN 46202

$\mathrm{Ph}$ (317) 274-3888, F (317) 321-0128

Author contribution: made significant revisions to manuscript

Michael Adjei, M.S.

Medical Student

Pediatric and Adolescent Comparative Effectiveness Research

Indiana University School of Medicine, Department of Pediatrics

$410 \mathrm{~W} .10^{\text {th }}$ Street, Suite 2000A, Indianapolis, IN 46202

$\mathrm{Ph}$ (571) 435-7533, F (317) 321-0128

Author contribution: review of manuscript; conducted research activities

Kathryn M. Haberlin-Pittz, MPH, CHES

Health Coach

Pediatric and Adolescent Comparative Effectiveness Research

Indiana University School of Medicine, Department of Pediatrics

$410 \mathrm{~W} .10^{\text {th }}$ Street, Suite 2000A, Indianapolis, IN 46202

$\mathrm{Ph}$ (317) 278-9641, F (317) 321-0128

Author contribution: review of manuscript; conducted research activities

Megan Agnew, MPH

Project Assistant

University of Wisconsin-Madison Department of Population Health Sciences

Warf Office Building, 610 Walnut Suite 707, Madison, WI 53726

$\mathrm{Ph}$ (812) 447-5145, F (317) 321-0128

Author contribution: conducted research activities

Tamara S. Hannon, M.D.

Professor of Pediatrics

Indiana University School of Medicine, Department of Pediatrics

Division of Pediatric Endocrinology and Diabetology

$410 \mathrm{~W} .10^{\text {th }}$ Street, Suite $2000 \mathrm{~A}$, Indianapolis, IN 46202

$\mathrm{Ph}$ (317) 944-3889, F (317) 321-0128

Author contribution: review of manuscript

This is the author's manuscript of the article published in final edited form as:

Yazel-Smith, L., El-Mikati, H. K., Adjei, M., Haberlin-Pittz, K. M., Agnew, M., \& Hannon, T. S. (2020). Integrating Diabetes Prevention Education Among Teenagers Involved in Summer Employment: Encouraging Environments for Health in Adolescence (ENHANCE). Journal of Community Health, 45(4), 856-861. https://doi.org/10.1007/s10900-020-00802-2 


\begin{abstract}
Introduction: Type 2 diabetes (T2D) in youth is a growing healthcare and public health concern. It is costly, and youth suffer from disabling and deadly comorbid conditions at a faster pace than adult onset. However, T2D is preventable. The population of obese youth at greatest risk for T2D is of minority race/ethnicity and socioeconomically disadvantaged background, which creates barriers to health promoting lifestyles. Despite being the first line of prevention efforts for T2D, efficacious behavioral lifestyle interventions are still lacking at the community level.
\end{abstract}

Methods: During the summers of 2016 and 2017, a study integrated obesity and diabetes prevention health education into TeenWorks summer employment program at Indy Urban Acres in Indianapolis, Indiana. Results were analyzed using paired sample t-tests.

Results: Participants $(\mathrm{N}=168)$ had a mean age of $15.8 \pm 0.7$ years, $61 \%$ female, $13 \%$ Hispanic, $80 \%$ Black. By the end of the intervention, physical activity $(\mathrm{p}=0.000)$ and prevention knowledge $(p=0.000)$ were significantly higher. Dietary intake $(p=0.204)$, self-efficacy $(p=0.58)$, food insecurity $(p=0.058)$ and depression screening scores $(p=0.809)$ were not significantly different.

Conclusion: In light of the continuing childhood obesity epidemic and increasing prevalence of prediabetes and T2D in youth, there is a pressing need to understand and reduce barriers to obesity and diabetes prevention in high-risk populations. This study demonstrated the feasibility of integrating obesity and T2D prevention health education into a teen summer employment program.

Keywords: youth, obesity, diabetes prevention, worksite 


\section{Introduction}

Pediatric obesity is on the rise in the United States with up to $18.5 \%$ of youth living with obesity, with a higher predilection to racial/ethnic minorities and socioeconomically disadvantaged groups (Hales, Carroll, Fryar, \& Ogden, 2017). Obesity in youth is associated with complications ranging from school problems(An, Yan, Shi, \& Yang, 2017; Wu, Chen, Yang, \& Li, 2017) to lifelong struggles with metabolic and cardiovascular diseases including early onset of type 2 diabetes (T2D)(Barshop, Francis, Schwimmer, \& Lavine, 2009; Dea, 2011; Tuomilehto, Seppa, \& Uusitupa, 2013). Obesity is seen in populations with prominent food insecurity(Castillo et al., 2012), limited access to safe places to exercise(Dias \& Whitaker, 2013) and to transportation (Barlow \& Ohlemeyer, 2006), as well as other barriers to engaging in behavioral lifestyle programming. Consequently, stakeholders face multiple issues when trying to implement behavioral lifestyle interventions at a community level.

Holding interventions at locations where adolescents already gather such as schools or workplace can prove to be an opportunity to engage at-risk adolescents in these interventions. School-based interventions can support healthy weight in youth by providing healthier food options and allocating time for physical activity in their curricula(Andrade et al., 2014). Engaging adolescents at their workplaces can also be helpful given that workplace provides a crucial setting for the development of self-definition, identification of social roles as well as improvement of problem solving skills, social competencies, and goal making(Mortimer, Zimmer-Gembeck, Holmes, \& Shanahan, 2002; Youniss, McLellan, Su, \& Yates, 1999). These skills are required to manage healthy weight, and hence, can be transferred to support an understanding of the importance of health. 
Encouraging Environments for Health in Adolescents (ENHANCE) was a program that we implemented over two summers to promote obesity and diabetes prevention within a teen summer employment program called TeenWorks. The primary aim of this study was to examine if offering a health education program to adolescents at a work site changed health behaviors.

\section{Methods}

A health education specialist (HES) and a medical student integrated the obesity and diabetes health education curriculum into 30-minute weekly sessions for the duration of the employment program by TeenWorks (TeenWorks.org). TeenWorks is a not-for-profit organization that offered a paid, six-week summer employment and college readiness program for high school students. TeenWorks offered two shifts per day: morning and an afternoon, both of which were given the ENHANCE curriculum. TeenWorks provided adolescent employees with meals, snacks and transportation to and from their designated community-based work site on a daily basis. Employees must be 15-18 years old, reside in Indianapolis, earn a minimum grade point average of 2.5, and be eligible for free or reduced lunch. The sessions were built into the workday at one work site, which was Indy Urban Acres (IUA), an eight-acre organic farm (http://www.cityparksalliance.org/why-urban-parks-matter/frontline-parks/parks/319) that cultivates fresh produce and donates $100 \%$ of its harvest to local food pantries and community members. As part of the work on the farm, adolescents are involved in approximately 3-hours of farm work per day, such as weeding, planting, digging, potting, and harvesting vegetable.

Prior to the study, teens took home a study information sheet for their parents'/legal guardians' review. Parental permission and teen assent were required to take part in the study. A research study staff member discussed the informed consent/assent document and the study procedures with the interested teens who provided written signature upon agreement to taking 
part in the study. All study documents were kept in locked cabinets in a secure research suite. Participants received $\$ 10.00$ gift cards for participation in the study at baseline and 6-week measures.

During the first summer only, heights and weights were measured to monitor any change in BMI during the 6-week period. Heights and weights were documented using calibrated electronic scales (Detecto; www.Detecto.com) and portable stadiometers (Seca; www.seca.com) at the baseline and 6-week measures. Shoes and other personal belongings (backpacks, cellphones) were removed prior to measuring height and weight. Given that the research study team did not expect any significant changes during the short time period of 6 weeks, BMI measures were not repeated during the second summer.

Participants completed a 31-question paper-and-pencil questionnaire given during the first week of the program and again during the final week (Appendix A). The questionnaire was comprised of seven demographic questions; five physical activity questions from the Youth Risk Behavior Surveillance System (CDC, 2015), 15 original nutrition questions to assess dietary intake, nutrition knowledge, and perceived self-efficacy of various nutrition behaviors, two food security questions(Hager et al., 2010), and 2 depression screening questions (PHQ-2)(Lowe, Kroenke, \& Grafe, 2005). Each participant took approximately 4 minutes to complete the survey. ENHANCE offered the following primary health messages: (a) reduction of consumption of sugary beverages, (b) increasing consumption of fruits and vegetables, (c) portion control and healthy snack strategies, and (d) increasing physical activity and decreasing sedentary behaviors during the teens' normal daily routine. The study was approved by the Indiana University Institutional Review Board (Protocol 1604487508).

\section{Theory}


Using the Social Ecological Model (SEM), ENHANCE was designed to affect interpersonal, intrapersonal, and community factors associated with obesity.

Interpersonal: ENHANCE was designed to affect the interpersonal level by building social support systems amongst adolescents working with TeenWorks and by creating social networks through their interaction with the health educator, a medical student, IUA staff members and peers. Building social support systems within the adolescent cohort was essential to reinforce the messages conveyed by ENHANCE given the importance of peer support when learning new habits.

Intrapersonal: ENHANCE was designed to change personal knowledge, attitudes and selfefficacy, which can eventually influence behaviors. The sessions focused on improving selfefficacy by making examples that addressed some of the adolescents' behaviors, while trying to keep it fun.

Community: Indy Urban Acres donates 100\% of its fresh produce to local food pantries and community organizations. The TW youth harvest the produce during their time on the farm. This activity connects the youth with the local community in a way that may not be afforded otherwise. Additionally, due to working at IUA, the availability of fresh fruits and vegetables for adolescents who participated in TeenWorks is much higher than that of some of their peers in the local community. Therefore, youth were more aware of access to produce issues that are prevalent in the community.

\section{Data Analysis}

Each individual's answers were scored per category: physical activity, dietary intake, knowledge and self-efficacy. These raw scores were then converted to a scaled score over 100. Means of the 
total scaled scores were compared using paired sample t-test using Statistical Package for Social Sciences version 25 (SPSS 25). 


\section{Results}

The study included 168 participants. The participants were adolescents with a mean age of $15.8 \pm 0.7$ years, majority female (61\%), and black race/ethnicity (80\%). Over both years, 144 participants completed the intervention with an $85 \%$ retention rate, which was due to youth dropping out of the summer employment program (Table 1).

The results of the questionnaires are presented in Table 2. At the level of behavioral changes, the participants maintained the same level of physical activity outside the farm when it comes to implementing toning exercises $(p=0.13)$ and being involved in school related physical education $(p=0.15)$. Similarly, the adolescents maintained comparable sedentary behaviors like watching TV $(p=0.38)$ and playing video games $(p=0.07)$. We documented however, that the participants reported increasing physical activity within the time frame of the study $(\mathrm{p}<0.01)$

ENHANCE participation was associated with an increased knowledge about healthy behaviors like the proper ration of vegetables in a meal $(\mathrm{p}<0.01)$, identifying that fruit juice is a major source of sugar $(\mathrm{p}<0.01)$ and that homemade meals are often healthier $(\mathrm{p}<0.01)$. However, scores did not improve when it comes to identifying that eating a healthy breakfast is important $(p=0.49)$ and that restaurant meals are higher in calories, fat and salt $(p=0.56)$

Dietary intake was not different after the program with participants having the same frequency of homemade meals $(p=0.71)$, fast food meals $(p=0.33)$, restaurant-meals $(p=0.10)$, having breakfast $(\mathrm{p}=0.25)$ or eating until they are too full $(\mathrm{p}=0.30)$. Moreover, self-efficacy did not change by the end of the intervention when it came to the perceived capacity to increase water intake instead of other sweetened beverages $(\mathrm{p}=0.95)$, having fruits with meals $(\mathrm{p}=0.78)$ or snacks $(p=0.17)$, having meals at home $(p=0.73)$, or eating breakfast daily $(p=0.25)$. 
Food security scores approached significance $(\mathrm{p}=0.05)$, while there was no significance found in depression scores $(\mathrm{p}=0.54)$. Results of the BMI measures in year one were not significant and are not presented here.

\section{Discussion}

Sugary drinks and added sugars are well-known contributors to the increase of childhood obesity(Ludwig, Peterson, \& Gortmaker, 2001) hence, interventions that can bridge this knowledge gap for adolescents should be encouraged. This study demonstrated the feasibility of integrating health education curriculum into a teen summer employment program for increasing participants' knowledge about nutritional value, most prominently identifying better nutritional choices and increasing physical activity. We documented, however, that even when the adolescents perceive the importance of eating healthier foods, in the absence of perceived selfefficacy, adolescents did not change their dietary intake. This is particularly interesting given that previous studies have successfully used youth empowerment as a sole intervention to decrease sugar-sweetened drinks(Wang, Otis, Rosal, Griecci, \& Lemon, 2019). Our results emphasize the importance of self-efficacy in the implementation of lifestyle changes. Youth at risk for obesity and type 2 diabetes often belong to lower socioeconomic status households(Nadeau et al., 2016). Hence, integrating health education curricula into employment settings can be a promising opportunity for high-risk youth especially when it engages them in diabetes prevention education while still earning a paycheck.

This study was limited by the small sample size, which affects the generalizability of our results. Moreover, the abbreviated time period did not allow for in-depth intervention with youth. Offering the intervention for more than 6-weeks might allow for additional significant outcomes. 
Youth employees were a captive audience in this study. Therefore, the outcomes only reflect data from a group that was present for another obligation.

\section{Conclusion}

ENHANCE, a 6-week summer program, is an efficacious intervention to increase physical activity and knowledge about healthier eating. However, it is not enough to change behaviors. The efficacy of similar but longer programs that address this issue should be investigated. There are no conflicts of interest to disclose. 


\section{References}

An, R., Yan, H., Shi, X., \& Yang, Y. (2017). Childhood Obesity and School Absenteeism: A Systematic Review and Meta-Analysis. Obesity Reviews, 18(12), 1412-1424. doi:10.1111/obr.12599

Andrade, S., Lachat, C., Ochoa-Aviles, A., Verstraeten, R., Huybregts, L., Roberfroid, D., . . . Kolsteren, P. (2014). A School-Based Intervention Improves Physical Fitness in Ecuadorian Adolescents: A Cluster-Randomized Controlled Trial. International Journal of Behavioral Nutrition and Physical Activity, 11, 153. doi:10.1186/s12966-014-0153-5

Barlow, S. E., \& Ohlemeyer, C. L. (2006). Parent Reasons for Nonreturn to a Pediatric Weight Management Program. Clin Pediatr (Phila), 45(4), 355-360. doi:10.1177/000992280604500408

Barshop, N. J., Francis, C. S., Schwimmer, J. B., \& Lavine, J. E. (2009). Nonalcoholic Fatty Liver Disease as a Comorbidity of Childhood Obesity. Pediatric health, 3(3), 271-281. doi:10.2217/phe.09.21

Castillo, D. C., Ramsey, N. L., Yu, S. S., Ricks, M., Courville, A. B., \& Sumner, A. E. (2012). Inconsistent Access to Food and Cardiometabolic Disease: The Effect of Food Insecurity. Curr Cardiovasc Risk Rep, 6(3), 245-250.

CDC, Centers for Disease Control and Prevention (2015). Youth Risk Behavior Survey. wWw.cdc.gov/YRBSS.

Dea, T. L. (2011). Pediatric Obesity \& Type 2 Diabetes. American Journal of Maternal/Child Nursing, 36(1), 42-48. doi:10.1097/NMC.0b013e3181fc06e7 
Dias, J. J., \& Whitaker, R. C. (2013). Black Mothers' Perceptions about Urban Neighborhood Safety and Outdoor Play for Their Preadolescent Daughters. J Health Care Poor Underserved, 24(1), 206-219. doi:10.1353/hpu.2013.0018

Hager, E. R., Quigg, A. M., Black, M. M., Coleman, S. M., Heeren, T., Rose-Jacobs, R., . . Frank, D. A. (2010). Development and Validity of a 2-Item Screen to Identify Families at Risk for Food Insecurity. Pediatrics, 126(1), e26. doi:10.1542/peds.2009-3146

Hales, C. M., Carroll, M. D., Fryar, C. D., \& Ogden, C. L. (2017). Prevalence of Obesity Among Adults and Youth: United States, 2015-2016. National Center for Health Statistics Data Brief(288), 1-8.

Lowe, B., Kroenke, K., \& Grafe, K. (2005). Detecting and Monitoring Depression with a Two-item Questionnaire (PHQ-2). J Psychosom Res, 58(2), 163-171. doi:10.1016/j.jpsychores.2004.09.006

Ludwig, D. S., Peterson, K. E., \& Gortmaker, S. L. (2001). Relation between Consumption of Sugar-Sweetened Drinks and Childhood Obesity: a Prospective, Observational Analysis. Lancet, 357(9255), 505-508. doi:10.1016/s0140-6736(00)04041-1

Mortimer, J. T., Zimmer-Gembeck, M. J., Holmes, M., \& Shanahan, M. J. (2002). The Process of Occupational Decision Making: Patterns during the Transition to Adulthood. Journal of Vocational Behavior, 61(3), 439-465. doi:https://doi.org/10.1006/jvbe.2002.1885 Nadeau, K. J., Anderson, B. J., Berg, E. G., Chiang, J. L., Chou, H., Copeland, K. C., . . Zeitler, P. (2016). Youth-Onset Type 2 Diabetes Consensus Report: Current Status, Challenges, and Priorities. Diabetes Care, 39(9), 1635-1642. doi:10.2337/dc16-1066 
Tuomilehto, H., Seppa, J., \& Uusitupa, M. (2013). Obesity and Obstructive Sleep Apnea--Clinical Significance of Weight Loss. Sleep Med Rev, 17(5), 321-329.

doi:10.1016/j.smrv.2012.08.002

Wang, M. L., Otis, M., Rosal, M. C., Griecci, C. F., \& Lemon, S. C. (2019). Reducing Sugary Drink Intake Through Youth Empowerment: Results from a Pilot-Site Randomized Study. Int J Behav Nutr Phys Act, 16(1), 58-58. doi:10.1186/s12966-019-0819-0

Wu, N., Chen, Y., Yang, J., \& Li, F. (2017). Childhood Obesity and Academic Performance: The Role of Working Memory. Frontiers in Psychology, 8, 611. doi:10.3389/fpsyg.2017.00611 Youniss, J., McLellan, J. A., Su, Y., \& Yates, M. (1999). The Role of Community Service in Identity Development: Normative, Unconventional, and Deviant Orientations. Journal of Adolescent Research, 14(2), 248-261. doi:10.1177/0743558499142006 